\title{
Self-assembled lecithin/chitosan nanoparticles for oral insulin delivery: preparation and functional evaluation
}

This article was published in the following Dove Press journal:

International Journal of Nanomedicine

24 February 2016

Number of times this article has been viewed

\section{Liyao Liu \\ Cuiping Zhou \\ Xuejun Xia \\ Yuling Liu}

State Key Laboratory of Bioactive Substance and Function of Natural Medicines, Beijing Key Laboratory of Drug Delivery Technology and Novel Formulations, Department of Pharmaceutics, Institute of Materia Medica, Chinese Academy of Medical Sciences \& Peking Union Medical College, Beijing, People's Republic of China
Correspondence: Yuling Liu State Key Laboratory of Bioactive Substance and Function of Natural Medicines, Beijing Key Laboratory of Drug Delivery Technology and Novel Formulations, Department of Pharmaceutics, Institute of Materia Medica, Chinese Academy of Medical Sciences \& Peking Union Medical College, I Xian Nong Tan Street, Beijing 100050,

People's Republic of China

$\mathrm{Tel}+861083160332$

Fax +86 1063167757

Email ylliu@imm.ac.cn
Purpose: Here, we investigated the formation and functional properties of self-assembled lecithin/chitosan nanoparticles (L/C NPs) loaded with insulin following insulin-phospholipid complex preparation, with the aim of developing a method for oral insulin delivery.

Methods: Using a modified solvent-injection method, insulin-loaded L/C NPs were obtained by combining insulin-phospholipid complexes with L/C NPs. The nanoparticle size distribution was determined by dynamic light scattering, and morphologies were analyzed by cryogenic transmission electron microscopy. Fourier transform infrared spectroscopy analysis was used to disclose the molecular mechanism of prepared insulin-loaded L/C NPs. Fast ultrafiltration and a reversed-phase high-performance liquid chromatography assay were used to separate free insulin from insulin entrapped in the L/C NPs, as well as to measure the insulin-entrapment and drug-loading efficiencies. The in vitro release profile was obtained, and in vivo hypoglycemic effects were evaluated in streptozotocin-induced diabetic rats.

Results: Our results indicated that insulin-containing L/C NPs had a mean size of $180 \mathrm{~nm}$, an insulin-entrapment efficiency of $94 \%$, and an insulin-loading efficiency of $4.5 \%$. Cryogenic transmission electron microscopy observations of insulin-loaded L/C NPs revealed multilamellar structures with a hollow core, encircled by several bilayers. In vitro analysis revealed that insulin release from L/C NPs depended on the L/C ratio. Insulin-loaded L/C NPs orally administered to streptozotocin-induced diabetic rats exerted a significant hypoglycemic effect. The relative pharmacological bioavailability following oral administration of L/C NPs was $6.01 \%$.

Conclusion: With the aid of phospholipid-complexation techniques, some hydrophilic peptides, such as insulin, can be successfully entrapped into L/C NPs, which could improve oral bioavailability, time-dependent release, and therapeutic activity.

Keywords: insulin-phospholipid complex, lecithin, chitosan, nanoparticles, oral delivery, streptozotocin-induced diabetes

\section{Introduction}

With the development of biotechnology, peptide-based pharmaceuticals have been introduced into clinical practice. ${ }^{1}$ Insulin, a major protein hormone secreted by pancreatic $\beta$-cells, plays a crucial role in the regulation of glucose metabolism and is used as a drug to control type-1 diabetes mellitus. Insulin is commonly administered by daily subcutaneous (sc) injections, leading to low patient compliance and unsatisfactory metabolic regulation. ${ }^{2}$ Oral insulin administration is more convenient, less invasive, and mimics physiological uptake via the hepatic route; ${ }^{3}$ therefore, various oral insulindelivery systems such as liposomes, ${ }^{4}$ emulsions,${ }^{5}$ microspheres,${ }^{6}$ and polymer-based nanoparticles (NPs) ${ }^{7,8}$ have been developed. However, the bioavailability of orally 
administered insulin preparations is not satisfactory because of their inherent instabilities in the gastrointestinal tract and inefficient absorption due to low permeability across biological membranes.

Chitosan is a natural polycationic polymer with mucoadhesive properties, which can facilitate protein absorption in the intestinal tract through opening tight junctions between epithelial cells. ${ }^{9,10}$ Chitosan can be obtained from chitin by alkaline deacetylation and has been used to prepare NPs by the ionotropic method ${ }^{11}$ or the emulsification solventdiffusion method. ${ }^{12}$ Significant progress has been achieved in synthesizing and purifying chitosan, as well as in applications of modified chitosan NPs for efficient oral insulin delivery. ${ }^{13,14}$ However, the above-mentioned methods have drawbacks, such as the requirement of chemical cross-linking agents and/or repeated washing and precipitation steps. Based on the development of the self-assembly technique, a novel self-assembled nanoparticulate system consisting of lecithin and chitosan was fabricated by the solvent-injection method. ${ }^{15}$ Through self-association, negatively charged phospholipids and positively charged chitosan formed stiff and stable nanostructures, which demonstrated good biocompatibility and biodegradability, excellent mucosal adhesiveness, and minimal cytotoxicity. ${ }^{16,17}$ This novel delivery system improved the bioavailability of model drugs and prolonged their retention times in the body. Several recent studies have investigated the efficiency of this novel nanocarrier in delivering various model drugs via different routes, including oral and transmucosal administration. ${ }^{18-21}$ However, it is believed that only hydrophobic molecules such as progesterone and natamycin can be entrapped into this self-assembled nanocarrier with high entrapment efficiency, whereas poor drug loading (DL) was observed for hydrophilic drugs. ${ }^{22}$

Insulin is a large, highly hydrophilic protein that cannot be loaded directly into chitosan-lecithin nanocarriers. However, results from a previous study demonstrated that insulin lipophilicity can be increased through chemical modifications or physical assemblage with phospholipids, which may facilitate its mucosal permeability and stability to enzymatic degradation. ${ }^{23}$ Because chemical modifications of high-molecular weight proteins often result in structural changes, insulin-phospholipid complexes are preferable. ${ }^{24}$ In our previous study, we developed a milder method to prepare insulin-phospholipid complexes with a high complexation rate of $\sim 100 \%$. Compared with insulin alone, the obtained insulin-phospholipid complexes had improved lipophilicity, and their solubility in n-octyl alcohol increased by $>40,000$-fold, while the biological activity of insulin was retained. ${ }^{25}$
In this study, we present a novel application of selfassembled lecithin/chitosan nanoparticles (L/C NPs) to entrap a hydrophilic therapeutic peptide with high molecular weight (insulin). We prepared insulin-loaded L/C NPs, which demonstrated high drug entrapment efficiency of phospholipid-complexed insulin. Insulin-entrapped L/C NPs were characterized in terms of their physicochemical properties, in vitro insulin release profile, and in vivo hypoglycemic effects in streptozotocin (STZ)-induced diabetic rats.

\section{Materials and methods Materials}

Recombinant human insulin was purchased from Dongbao Enterprise Group Co. Ltd. (Jilin, People’s Republic of China), lecithin (70\% phosphatidylcholine/30\% phosphatidylethanolamine, Lipoid S75) was obtained from Shanghai Toshisun Biology \& Technology Co. Ltd. (Shanghai, People's Republic of China), chitosan (90\%-95\% deacetylated) was from Fluka (Buchs, Sweden), and STZ was from Sigma-Aldrich (St Louis, MO, USA). Phosphate buffer solution (PBS; $\mathrm{pH} 7.4$, without calcium or magnesium, Hyclone) was from Thermo Scientific (Waltham, MA, USA). Other chemicals and solvents were of analytical or chromatography grade.

\section{Animals}

Male Sprague Dawley rats were purchased from the Experimental Animal Center of National Institutes for Food and Drug Control. All rats were raised in the Institute of Materia Medica, Chinese Academy of Medical Sciences \& Peking Union Medical College (Beijing, People's Republic of China). All of the experiments were performed with the approval of the Laboratory Animal Care and Use Committee of Peking Union Medical College.

\section{Preparation of insulin-phospholipid complexes}

The preparation of insulin-phospholipid complexes was performed as described previously. ${ }^{25}$ Briefly, insulin (10 mg) was dissolved in $0.1 \%$ trifluoroacetic acid-methanol $(1 \mathrm{~mL})$, and Lipoid S75 (100 mL) was dissolved in dichloromethane $(10 \mathrm{~mL})$. After mixing insulin and Lipoid S75 together, the organic solvents were removed by rotary evaporation in a vacuum. Nitrogen was then flushed into the flask to remove the organic solvents thoroughly, and the flask was left in the vacuum oven $\left(37^{\circ} \mathrm{C}\right)$ overnight. On the following day, the resulting complex was stored at $-20^{\circ} \mathrm{C}$.

\section{Preparation of insulin-loaded L/C NPs}

Insulin-loaded L/C NPs were prepared using a modified solvent-injection method. Briefly, chitosan was dissolved 
in $1 \%(\mathrm{v} / \mathrm{v})$ acetic acid to obtain a $1 \%(\mathrm{w} / \mathrm{v})$ chitosan stock solution, which was used to prepare various dilutions in distilled water. Then, $8 \mathrm{~mL}$ of each insulin-phospholipid complex in ethanol (insulin-phospholipid complex concentration, $27.5 \mathrm{mg} / \mathrm{mL}$, which contains $25 \mathrm{mg}$ lecithin and $2.5 \mathrm{mg}$ insulin in $1 \mathrm{~mL}$ liquid) was injected through a syringe needle $(0.7 \mathrm{~mm}$, inner diameter) into $92 \mathrm{~mL}$ of each chitosan dilution to achieve lecithin/chitosan (L/C) ratios ranging from 10:0 to 40:1 (w/w), and stirred to obtain drugloaded L/C NPs.

For comparison purposes, insulin-free (empty) L/C NPs were fabricated by substituting insulin-phospholipid complexes with lecithin solution in ethanol using the same procedure. In addition, insulin-loaded S75 phospholipid vesicles were prepared by injecting insulin-lecithin complexes into distilled water.

\section{Characterization of particle size, surface charge, and morphology}

Dynamic light scattering (Nicomp 380 ZLS, Particle Size System; Santa Barbara, CA, USA) was used to measure the average particle size. Prior to analysis, samples were diluted in distilled water filtered through a $0.22 \mu \mathrm{m}$ membrane to avoid multiple scattering. Measurements were performed at room temperature using a scattering angle of $90^{\circ}$. The zeta potential was determined by measuring the Doppler electrophoretic laser shift at $28^{\circ} \mathrm{C}$ and analyzing light scattering at $-14^{\circ} \mathrm{C}$, using a Nicomp 380 ZLS instrument. The morphology of prepared NPs and vesicles was evaluated by cryogenic transmission electron microscopy (TECNAI 20; Philips, Amsterdam, the Netherlands). NP suspensions were deposited on polycarbonate grids and rapidly cooled with liquid nitrogen; the obtained thin, vitrified aqueous films were used for morphological observations.

\section{FT-IR analysis}

Fourier transform infrared spectroscopy (FT-IR) analysis (Nicolet 5700; Thermo Electron Corporation, Waltham, MA, USA) was performed using lyophilized NPs to study whether insulin-phospholipid complexes dissociated during the preparation of insulin-loaded L/C NPs. Spectra were collected at $20^{\circ} \mathrm{C}$ in the scanning range of $4,000-400 \mathrm{~cm}^{-1}$.

\section{Determination of insulin loading}

The amount of total insulin in suspension was measured by dissolving NP suspensions in $\mathrm{pH}$ 7.4 PBS (without calcium or magnesium, Hyclone; Thermo Scientific). Briefly, 1\% Triton X100 was added to $2 \mathrm{~mL}$ of NP suspension. Subsequently, the solution was adjusted to a volume of $10 \mathrm{~mL}$ by $\mathrm{pH}$ 7.4 PBS. Then, a reversed-phase high-performance liquid chromatography (RP-HPLC) assay was performed to measure the amount of insulin in the solvent.

The encapsulation efficiency (EE) of insulin-loaded L/C NPs was measured using a fast ultrafiltration method. Briefly, $1 \mathrm{~mL}$ of each prepared NP suspension was placed in a centrifugal filter tube (Amicon Ultra-4 centrifugal devices, 100K NMWL; Millipore, Billerica, MA, USA) and centrifuged at 4,000 rpm for 20 minutes to separate free and entrapped insulin in NPs. After ultrafiltration, the RP-HPLC assay was used to measure the amount of insulin in ultrafiltrates with Agilent Technologies 1200 series HPLC system (Agilent, Santa Clara, CA, USA) and a 300SB-C8 column (4.6×250 mm, $5 \mu \mathrm{m}$, Agilent). The following conditions according to the China Pharmacopeia (2010) were used mobile phase: $0.1 \mathrm{M}$ sulfate buffer/acetonitrile (73:27, v/v), flow rate: $1 \mathrm{~mL} / \mathrm{min}$, ultraviolet detection: $214 \mathrm{~nm}$, injection volume: $20 \mu \mathrm{L}$, and column oven temperature: $40^{\circ} \mathrm{C}$. Then, $0.1 \mathrm{M}$ sulfate buffer containing $0.1 \mathrm{M}$ sodium sulfate and trifluoroacetic acid was added to adjust the $\mathrm{pH}$ value to 2.3. Each sample was analyzed in triplicate.

L/C NP EE and DL efficiency were calculated according to the following equations: ${ }^{19}$

$$
\begin{aligned}
& \mathrm{EE}=\frac{W_{\text {total insulin }}-W_{\text {free insulin }}}{W_{\text {total insulin }}} \times 100 \% \\
& \mathrm{DL}=\frac{W_{\text {total insulin }}-W_{\text {free insulin }}}{W_{\text {total NPs }}} \times 100 \%
\end{aligned}
$$

where $W_{\text {total insulin }}$ is the total amount of insulin in NP suspension, $W_{\text {free insulin }}$ is the amount of insulin in the filtrate solvent, and $W_{\text {total NPs }}$ is the amount of NPs measured after freeze-drying.

\section{In vitro insulin release}

The release profile of insulin from NPs was determined in PBS adjusted to $\mathrm{pH} 1.2$ or $\mathrm{pH} 6.8$, in the absence of added enzymes. In each case, $1.0 \mathrm{~mL}$ insulin-loaded L/C NP suspension (insulin concentration, $0.1 \mathrm{mg} / \mathrm{mL}$ ) was transferred into a centrifugal filter tube and centrifuged at 4,000 rpm for 20 minutes. Next, the ultrafiltrate was collected and dispersed into $10 \mathrm{~mL}$ release medium ( $\mathrm{pH} 1.2$ or $\mathrm{pH}$ 6.8). In vitro drugrelease tests were performed in a shaking water bath at $37^{\circ} \mathrm{C}$ and $50 \mathrm{rpm}$. At different time intervals, a $500 \mu \mathrm{L}$ aliquot was withdrawn, centrifuged at 3,000 rpm for 20 minutes, and the supernatant containing free insulin was collected and measured using the RP-HPLC assay. Each experiment was performed in triplicate. 


\section{In vivo hypoglycemic effect of insulin- loaded L/C NPs}

The pharmacological bioactivity of insulin delivered by orally administered L/C NPs was evaluated in diabetesmodel rats by assessing the hypoglycemic effect. All animal experiments were performed in accordance with principles of laboratory animal care, and approved by the Institutional Animal Ethical Committee of Beijing (People's Republic of China).

Before animal experiments were initiated, male Sprague Dawley rats were acclimated in an animal facility for 2 weeks. Diabetes was induced in rats with STZ $(60 \mathrm{mg} / \mathrm{kg}$ in citrate buffer, $\mathrm{pH}$ 4.2), which was administrated by intraperitoneal injection. After blood glucose levels were stabilized for 1 week, STZ-induced diabetic rats $(200 \pm 20 \mathrm{~g}$, blood glucose levels $\geq 16.7 \mathrm{mM}$ ) were fasted overnight and randomly divided into four groups ( $\mathrm{n}=3$ in each group). Group 1 was the control group, which received oral administration of insulin solution $(60 \mathrm{IU} / \mathrm{kg}$ ) by gavage. Group 2 was the positive control group, which was treated with sc injections of insulin in PBS (2 IU/kg). Groups 3 and 4 received insulinloaded L/C NPs at doses of 40 and $60 \mathrm{IU} / \mathrm{kg}$, respectively, by gavage. Blood samples were collected from the tail veins at $0,0.5,1,2,4,6,8,10$, and 12 hours after administration, and blood glucose levels were measured using a Blood Analyzer (ONETOUCH Ultra; Johnson \& Johnson, New Brunswick, NJ, USA).

The relative pharmacological bioavailability $\left(F_{\mathrm{p}}\right)$ of insulin after oral administration was calculated according to the following equation: ${ }^{26}$

$$
F_{\mathrm{p}}=\frac{\mathrm{AAC}_{\text {oral }} \times \text { Dose }_{\text {sc }}}{\mathrm{AAC}_{\text {sc }} \times \text { Dose }_{\text {oral }}} \times 100 \%
$$

where Dose $\mathrm{oral}_{\text {and }}$ anose $\mathrm{sc}_{\mathrm{sc}}$ are the insulin doses administered orally or subcutaneously, respectively, and AAC is the area above the curve of the reduction in blood glucose level over time.

\section{Statistical analysis}

The data were expressed as the mean \pm standard deviation and analyzed by one-way analysis of variance. Differences were considered statistically significant at $P<0.05$.

\section{Results}

\section{Preparation of insulin-loaded L/C NPs}

In this study, insulin-loaded L/C NPs were prepared to investigate their characteristics and drug EEs. First, we studied the conditions of insulin loading into L/C NPs. Preliminary experiments showed that the $\mathrm{pH}$ of chitosan solution affected the EE of insulin in NPs. To determine the optimal $\mathrm{pH}$ for insulin loading into $\mathrm{L} / \mathrm{C} \mathrm{NPs}$, an $\mathrm{L} / \mathrm{C}$ ratio of $20: 1(\mathrm{w} / \mathrm{w})$ was used, and the $\mathrm{pH}$ of the chitosan solution during L/C NP preparation was varied by extra addition of glacial acetic acid or $1 \mathrm{~N} \mathrm{NaOH}$. As shown in Table 1, the insulin EE increased in parallel with $\mathrm{pH}$ increases up to the isoelectric point of recombinant human insulin ( $\mathrm{pI}=5.4$ ). In fact, as the chitosan used in experiments would precipitate when the $\mathrm{pH}$ exceeded 6.5 , further studies using higher $\mathrm{pH}$ values of the chitosan solution were not performed. These results were consistent with previous results. ${ }^{27}$

Next, we determined the optimal $\mathrm{L} / \mathrm{C}$ ratio, using the selected $\mathrm{pH}$ value (5.4) for the chitosan solution and differing $\mathrm{L} / \mathrm{C}$ ratios $(10: 0,5: 1,10: 1,20: 1$, and 40:1) during insulin loading. As shown in Table 2, the $\mathrm{L} / \mathrm{C}$ ratio affected particle sizes, zeta potentials, and insulin EEs. In our experiments, insulin did not form phospholipid complexes with other lecithin types, such as S45 (40\% phosphatidylcholine/60\% phosphatidylethanolamine, Lipoid S45). Thus, we did not study L/C NPs with other lecithin types.

For comparison purposes, phospholipid vesicles loaded with $10 \mathrm{mg}$ insulin were prepared without chitosan (Table 2; $\mathrm{L} / \mathrm{C}$ ratio, 10:0). Compared with insulin-loaded L/C NPs, these insulin-loaded lecithin vesicles had a narrow size distribution and negative zeta potentials, whereas NPs prepared with the L/C ratios 10:1 and 20:1 had the highest insulin

Table I Influence of the $\mathrm{pH}$ of chitosan solution on the characteristics of insulin-loaded L/C NPs ${ }^{\mathrm{a}}$

\begin{tabular}{|c|c|c|c|c|c|}
\hline Formulation & $\mathrm{pH}$ of CS solution & EE (\%) ${ }^{b}$ & DL (\%) ${ }^{b}$ & Size $(\mathrm{nm})^{\mathrm{b}}$ & Zeta potential $(\mathrm{mV})^{\mathrm{b}}$ \\
\hline Al & 2.0 & $|8.0| \pm 0.72$ & $0.67 \pm 0.04$ & $128.33 \pm 9.45$ & $22.48 \pm 2.14$ \\
\hline $\mathrm{A} 2$ & 3.0 & $66.25 \pm 1.89$ & $2.16 \pm 0.21$ & $149.14 \pm 5.88$ & $23.56 \pm 1.97$ \\
\hline A3 & 4.0 & $83.04 \pm 1.66$ & $3.3 I \pm 0.1 I$ & $|77.36 \pm| \mid .07$ & $21.87 \pm 2.05$ \\
\hline A4 & 5.0 & $90.49 \pm 1.06$ & $3.8 I \pm 0.16$ & $168.27 \pm 7.35$ & $24.24 \pm 3.47$ \\
\hline A5 & 5.5 & $94.69 \pm 0.20$ & $4.60 \pm 0.16$ & $180.30 \pm 15.92$ & $24.40 \pm 1.75$ \\
\hline A6 & 6.0 & Floccules & Floccules & Floccules & Floccules \\
\hline
\end{tabular}

Notes: ${ }^{\text {TT }}$ The L/C ratio of $20: I$ was used. ${ }^{b}$ The data are presented as the mean $\pm S D(n=3)$.

Abbreviations: L/C NPs, lecithin/chitosan nanoparticles; CS, chitosan; EE, entrapment efficiency; DL, drug loading; SD, standard deviation. 
Table 2 Influence of different lecithin-to-chitosan ratios on main characteristics of insulin-loaded L/C NPs

\begin{tabular}{llllll}
\hline Formulation & L/C ratio & EE $(\%)^{\mathrm{a}}$ & DL $(\%)^{\mathrm{a}}$ & Size $(\mathrm{nm})^{\mathrm{a}}$ & ${\text { Zeta potential }(\mathrm{mV})^{\mathrm{a}}}^{\mathrm{a}}$ \\
\hline BI & $10: 0$ & $78.90 \pm 6.12$ & $9.37 \pm 0.87$ & $43.38 \pm 23.30$ & $-21.49 \pm 2.01$ \\
B2 & $5: 1$ & $89.03 \pm 1.55$ & $5.75 \pm 1.79$ & $125.05 \pm 22.16$ & $22.36 \pm 0.72$ \\
B3 & $10: 1$ & $94.32 \pm 0.34$ & $8.82 \pm 0.18$ & $159.42 \pm 7.17$ & $24.97 \pm 2.41$ \\
B4 & $20: 1$ & $94.69 \pm 0.20$ & $4.60 \pm 0.35$ & $180.12 \pm 15.72$ & $24.40 \pm 1.75$ \\
B5 & $40: 1$ & $93.10 \pm 0.44$ & $2.61 \pm 0.10$ & $202.08 \pm 9.00$ & $20.02 \pm 1.22$ \\
\hline
\end{tabular}

Note: ${ }^{\text {TT }}$ The data are presented as the mean \pm SD $(n=3)$.

Abbreviations: L/C NPs, lecithin/chitosan nanoparticles; L/C, lecithin/chitosan; EE, entrapment efficiency; DL, drug loading; SD, standard deviation.

EEs (Table 2). Thus, the optimal conditions for insulin loading in L/C NPs were $\mathrm{L} / \mathrm{C}$ ratios of $10: 1$ and 20:1, and a chitosan solution $\mathrm{pH}$ of 5.4 .

\section{Morphological characterization}

Cryogenic transmission electron microscopy analysis of insulin-loaded L/C NPs (L/C ratio, 20:1) revealed multilamellar structures with a hollow core circled by multiple bilayers (Figure 1A). In contrast, the morphology of the insulin-loaded phospholipid vesicles (L/C ratio, 10:0) was significantly different, showing a typical shell-core structure (Figure 1B).

\section{FT-IR analysis}

The FT-IR spectra of chitosan, insulin, lecithin, insulinphospholipid complexes, and insulin-loaded L/C NPs (L/C ratio, 20:1) are shown in Figure 2. Compared to our previous results, ${ }^{25}$ the characteristic absorption peaks of insulin at $1,660 \mathrm{~cm}^{-1}(v[\mathrm{CO}])$ and $1,520 \mathrm{~cm}^{-1}(\delta[\mathrm{NH}])$ were shifted to shorter wavelengths (blue shifted), whereas the absorption peak typical for phospholipids demonstrated a slight red shift (from $1,238.9 \mathrm{~cm}^{-1}$ to $1,235.5 \mathrm{~cm}^{-1}, v[\mathrm{P}=\mathrm{O}]$ ). In the spectrum of insulin-loaded L/C NPs, the characteristic absorption peaks of insulin did not shift to their original wavelengths, indicating that the insulin-phospholipid complexes did not dissociate during the preparation of insulinloaded L/C NPs.

\section{In vitro insulin release from L/C NPs}

As insulin dissolved in solution can be easily digested by enzymes and therefore cannot be measured, insulin release profiles from lecithin-chitosan self-assembled NPs were determined in simulated gastric fluid (SGF) and simulated intestinal fluid (SIF), without enzymes. As shown in Figure 3, the time-dependent release of insulin from L/C NPs was affected by the $\mathrm{L} / \mathrm{C}$ ratio. Insulin-loaded L/C NPs with an L/C ratio of 20:1 clearly demonstrated lower drug release, compared to those with an L/C ratio of 10:1.

$\mathrm{L} / \mathrm{C}$ NPs with $20: 1 \mathrm{~L} / \mathrm{C}$ ratio released $\sim 20 \%-30 \%$ of the loaded insulin over 2 hours in SGF and 20\% over 12 hours in SIF. However, L/C NPs with 10:1 L/C ratio released $50 \%-60 \%$ of the loaded insulin in the first 2 hours in SGF and
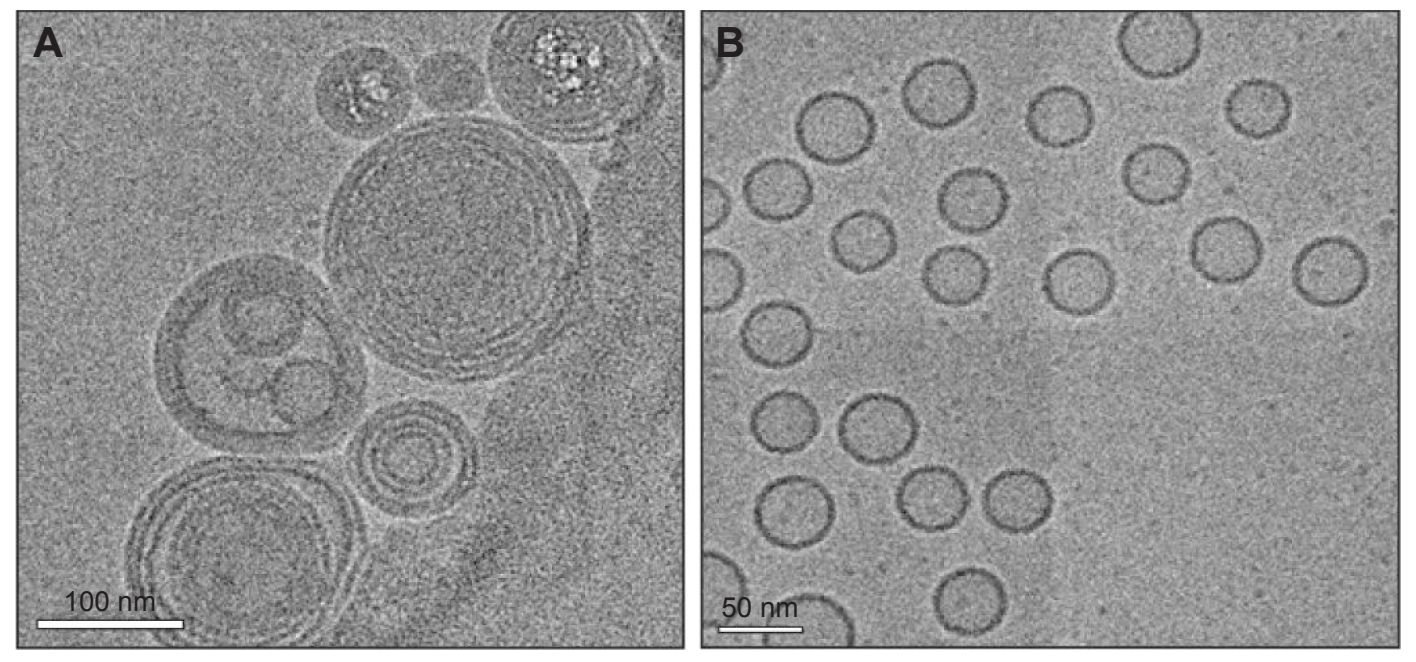

Figure I Morphology of insulin-loaded nanoparticles.

Notes: Cryogenic transmission electron microscopy images of insulin-loaded lecithin/chitosan nanoparticles (L/C ratio, 20:I) (A) and insulin-loaded vesicles (L/C ratio, I0:0) (B). Abbreviation: L/C, lecithin/chitosan. 


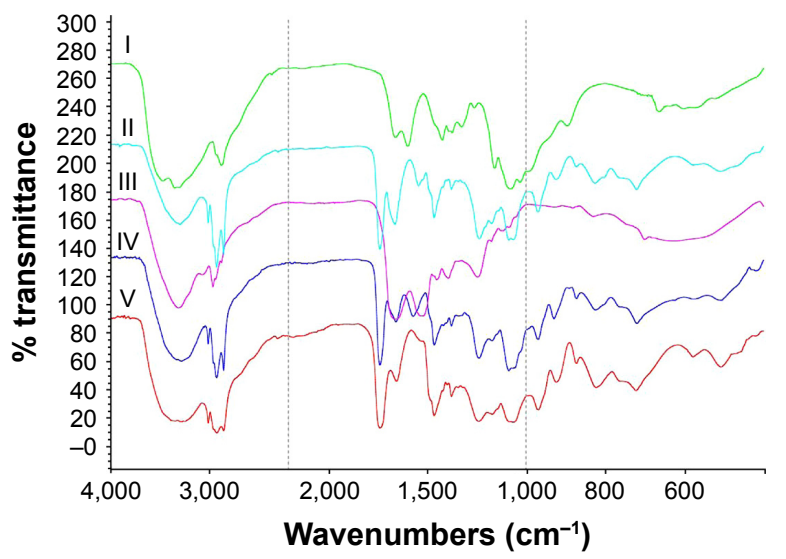

Figure 2 Fourier transform infrared spectroscopy analysis of the components of insulin-loaded L/C NPs.

Notes: I: chitosan, II: insulin-lecithin complex, III: insulin, IV: insulin-loaded L/C NPs (L/C ratio of $20: I$ ), and $V$ : lecithin.

Abbreviations: L/C NPs, lecithin/chitosan nanoparticles; L/C, lecithin/chitosan.

$50 \%$ after 12 hours in SIF. These results clearly indicated that insulin-loaded NPs with an L/C ratio of 20:1 demonstrated slower insulin release than those with a 10:1 ratio. Therefore, the former L/C NPs were used to test the hypoglycemic effect of insulin in vivo. In addition, insulin-loaded S75 lecithin vesicles dissociated and precipitated in both SGF and SIF.

\section{In vivo hypoglycemic effect of insulin- loaded L/C NPs}

To determine the hypoglycemic effect of insulin-loaded $\mathrm{L} / \mathrm{C}$ NPs, they were orally administered to diabetic rats after overnight fasting, and blood samples were analyzed for blood glucose levels. As shown in Figure 4, significant difference in blood glucose reduction between rats receiving free insulin (Group 1) and insulin-loaded L/C NPs (Groups 3 and 4) was observed until the end of the experiment $(P<0.05)$.
Glucose levels barely changed within 12 hours in Group 1, whereas a hypoglycemic effect in Group 4 was evident.

After sc injection of insulin, blood glucose was reduced by $85 \%$ of the initial level after 2 hours; this hypoglycemic effect was observed for up to 5 hours, after which it subsided. Orally administered insulin-loaded L/C NPs demonstrated slower but prolonged blood glucose reduction, which was maximal at 4 hours $(47 \%$ and $65 \%$ decrease by 40 and $60 \mathrm{IU} / \mathrm{kg} \mathrm{L} / \mathrm{C}$ NPs, respectively) and lasted for 12 hours. These results indicate that insulin-loaded L/C NPs provided gradual long-lasting insulin delivery into the blood and that, considering the results of in vitro release experiments, the insulin entrapped in the NPs was released slowly and thus prolonged the hypoglycemic effects. Table 3 shows that the pharmacological bioavailability $\left(F_{\mathrm{p}}\right)$ of insulin significantly increased when delivered by L/C NPs $(6.01 \%$ versus $0.53 \%$ for insulin solution orally administered, $P<0.05$ ).

\section{Discussion}

In this study, insulin-loaded L/C NPs with high drug EE were successfully prepared and analyzed for physicochemical properties and hypoglycemic effects in an experimental rat model of diabetes.

In previous studies, self-assembled L/C NPs have been used to deliver lipophilic drugs; however, some lipophilic compounds showed low solubility in methanol or ethanol with lecithin, and solubilizing agents such as tocopheryl polyethylene glycol succinate and isopropyl myristate were necessary. ${ }^{28}$ Nevertheless, poor hydrophilic DL into L/C NPs $(<1 \%)$ has been observed. ${ }^{15,22}$

Pure insulin is insoluble in absolute ethanol; however, we showed that insulin in complex with phospholipids had

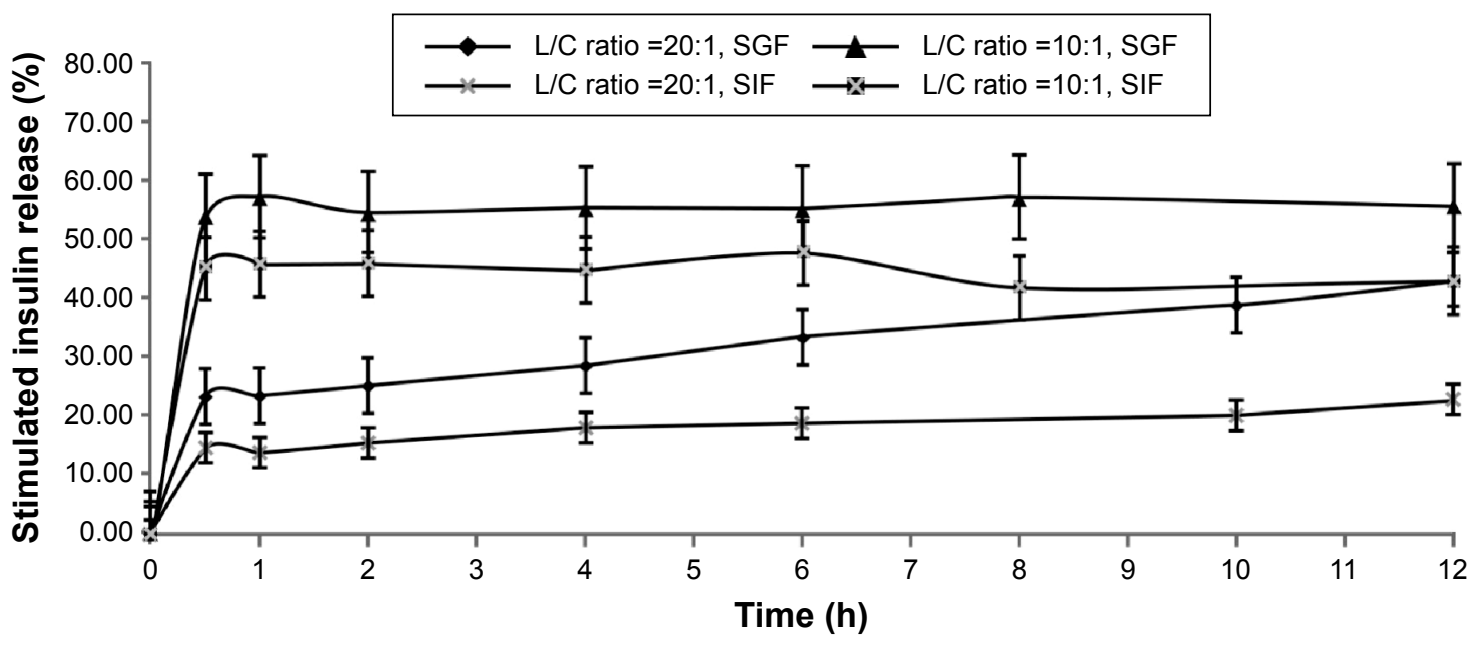

Figure 3 In vitro time-dependent release profiles of insulin-loaded lecithin/chitosan nanoparticles with different L/C ratios.

Note: The data are presented as the mean \pm SD $(n=3)$.

Abbreviations: L/C, lecithin/chitosan; SD, standard deviation; SGF, simulated gastric fluid; SIF, simulated intestinal fluid. 


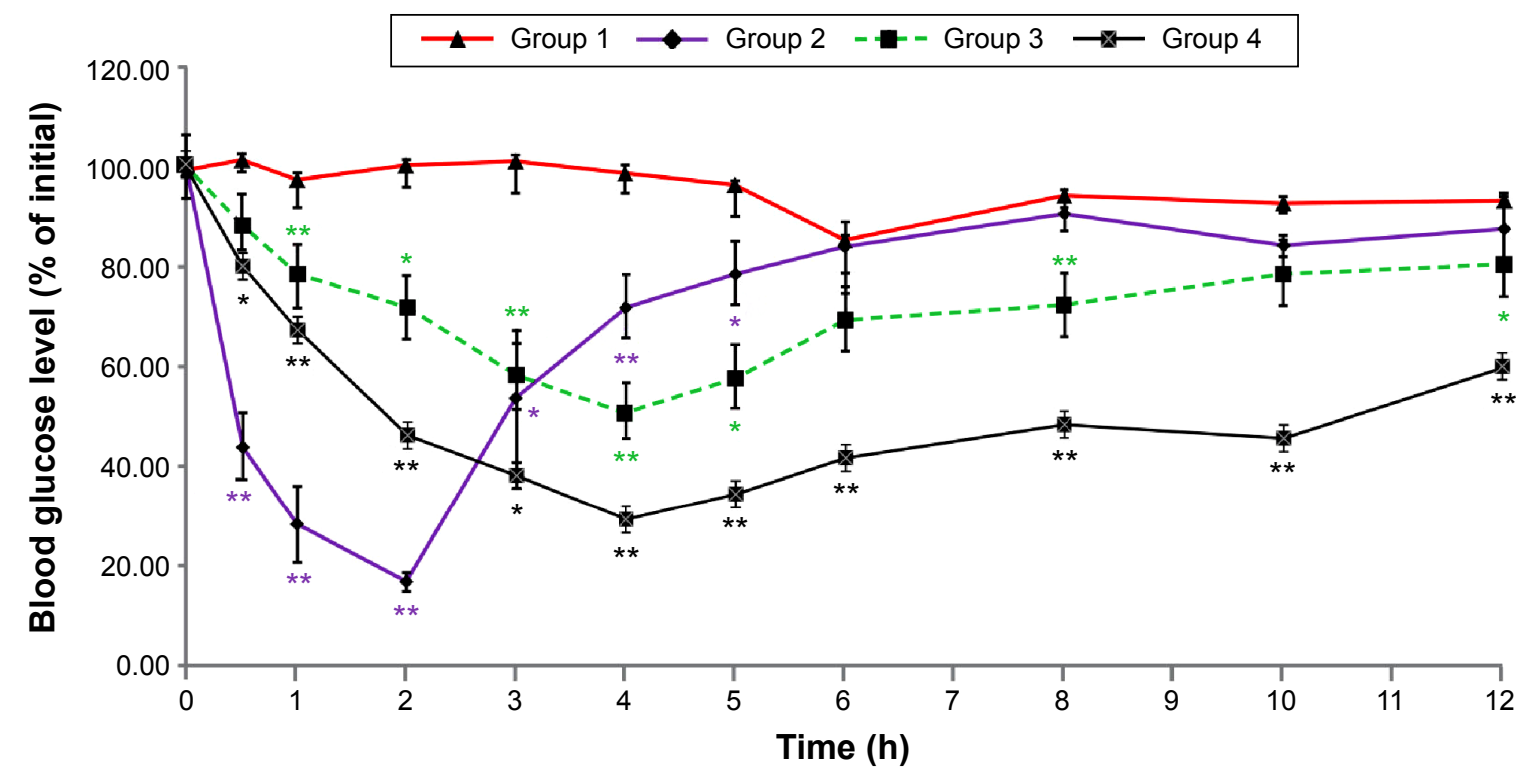

Figure 4 Time-dependent reduction in blood glucose levels in diabetic rats.

Notes: Streptozotocin-treated rats were administered insulin at the dose of $60 \mathrm{IU} / \mathrm{kg}$ by gavage (Group I) or 2 IU/kg subcutaneously (Group 2), or insulin-loaded lecithin/ chitosan nanoparticles (L/C ratio, 20:I) at the dose of $40 \mathrm{IU} / \mathrm{kg}$ (Group 3) or $60 \mathrm{IU} / \mathrm{kg}$ (Group 4) by gavage. The data are presented as the mean \pm SD ( $=3$ ); $* P<0.05$ and $* * P<0.01$ versus Group I.

Abbreviations: L/C, lecithin/chitosan; SD, standard deviation.

increased solubility in ethanol, which enabled successful encapsulation in L/C NPs with high entrapment efficiency ( $94 \%)$. Our results suggest that phospholipid complexation may widen the potential clinical applications for L/C NPs because drug-phospholipid complexes soluble in ethanol or methanol could be used to prepare drug-loaded L/C NPs for successful drug delivery and appropriate time-dependent release.

We determined that the $\mathrm{L} / \mathrm{C}$ ratio influenced particle size and the zeta potential, whereas the $\mathrm{pH}$ of the chitosan solution affected insulin-entrapment efficiency in NPs. Different combinations of insulin with lecithin were not tested because we previously determined that an insulin-phospholipid ratio of 10:1 was optimal for obtaining stable insulin-phospholipid complexes, ${ }^{25}$ which was used in the current experiments. Under these conditions, we obtained a maximal insulinloading rate of $10 \%$.

Table $3 \mathrm{AAC}_{0 \rightarrow 12 \mathrm{~h}}$ and $F_{\mathrm{p}}$ of insulin-loaded L/C NPs and free insulin

\begin{tabular}{lll}
\hline Formulation & AAC $_{0 \rightarrow 12 \mathrm{~h}}$ & $\boldsymbol{F}_{\mathrm{p}}(\%)$ \\
\hline Insulin solution (2 IU/kg, sc) & $344.29 \pm 13.42$ & 100.00 \\
Insulin solution (60 IU/kg, gavage) & $55.1 \mathrm{I} \pm 21.56$ & 0.53 \\
Insulin-loaded L/C NPs (L/C ratio of 20:I, & $621.09 \pm 8.85$ & $6.0 I^{* *}$ \\
$60 \mathrm{IU} / \mathrm{kg}$, gavage) & & \\
\hline
\end{tabular}

Notes: $A_{A C} C_{0 \rightarrow 12 h}$ data are presented as the mean $\pm S D(n=3)$. ${ }^{* * P}<0.01$ versus insulin solution administered by gavage.

Abbreviations: AAC, area above the curve; $F_{\mathrm{p}}$, pharmacological bioavailability; L/C NPs, lecithin/chitosan nanoparticles; sc, subcutaneous; L/C, lecithin/chitosan; SD, standard deviation.
Furthermore, the $\mathrm{L} / \mathrm{C}$ ratio affected in vitro time-dependent release of insulin from L/C NPs. In all formulations, we used an excess amount of chitosan..$^{22}$ The increase in lecithin concentration strengthened the electrostatic interaction in NPs and increased the number of bilayers, ensuring slow release of insulin entrapped in multilayered NPs. Thus, stronger electrostatic interactions can account for differences between NPs prepared with different $\mathrm{L} / \mathrm{C}$ ratios.

Finally, we developed a self-assembled oral insulindelivery system, which has several advantages over previous approaches. Specifically, insulin-loaded L/C NPs could be obtained without the use of cross-linking agents such as tripolyphosphate ${ }^{29}$ or glutaraldehyde ${ }^{26}$ through a simpler preparation procedure, which did not require homogenization or repeated washing and precipitation as done in previous studies. ${ }^{30,27}$ Furthermore, the EE of self-assembled insulinloaded L/C NPs was much higher than that of other delivery systems.

Although insulin-lecithin complexes could be selfassembled into L/C NPs, in vivo experiments with diabetic rats did not demonstrate a significant improvement in pharmacological bioavailability of insulin, compared with previous findings. ${ }^{4,7,30,31}$ Another limitation of this study is that pharmacokinetic/pharmacodynamic analyses to compare insulin bioavailability after oral and sc administration were not performed. Further research is required to address these issues.

It has been reported that improved oral bioavailability of NPs and protection from proteolytic degradation in the 
gastrointestinal tract could be helpful in reducing the insulin burst release. ${ }^{32,33}$ Relevant approaches include enteric coating and/or modification of NP surfaces. Thus, Eudragit S100 and HP-55 have demonstrated potential as enteric coatings for insulin-loaded L/C NPs. ${ }^{34,35}$ In our preliminary studies, Eudragit S100 microspheres provided a 5\% decrease in the initial burst release of insulin in SGF during the first 2 hours (data not shown). In studies of insulin release using orally administered L/C NPs, parameters such as the serum insulin level should be analyzed to assess drug absorption and to accurately determine its relative bioavailability.

\section{Conclusion}

In the present study, we show that large hydrophilic peptides such as insulin can be successfully loaded into self-assembled L/C NPs after complexation with phospholipids. Using this approach, a high insulin-entrapment efficiency of $\sim 94 \%$ was achieved, and insulin-containing L/C NPs of $\sim 180 \mathrm{~nm}$ in size were obtained. Insulin encapsulation into multilayered L/C NPs provided improved insulin release after oral administration, which resulted in the reduction of blood glucose levels in diabetic rats. Our findings suggest that L/C NPs can be successfully used for the delivery of hydrophilic drugs.

\section{Acknowledgments}

This study was supported by the Fundamental Research Funds for the Central Universities, the PUMC Youth Fund (Grant No 3332015044) from the Chinese Academy of Medical School and Peking Union Medical School, and the National Mega Project for Innovative Drugs (Grant No 2012ZX09301002-001) of the Chinese government.

\section{Disclosure}

The authors report no conflicts of interest in this work.

\section{References}

1. Sato AK, Viswanathan M, Kent RB, Wood CR. Therapeutic peptides: technological advances driving peptides into development. Curr Opin Biotechnol. 2006;17(6):638-642.

2. Saaddine JB, Cadwell B, Gregg EW, et al. Improvements in diabetes processes of care and intermediate outcomes: United States, 1988-2002. Ann Intern Med. 2006;144(7):465-474.

3. Heinemann L, Jacques Y. Oral insulin and buccal insulin: a critical reappraisal. J Diabetes Sci Technol. 2009;3(3):568-584.

4. Zhang X, Qi J, Lu Y, He W, Li X, Wu W. Biotinylated liposomes as potential carriers for the oral delivery of insulin. Nanomedicine. 2014; 10(1):167-176.

5. Morishita M, Matsuzawa A, Takayama K, Isowa K, Nagai T. Improving insulin enteral absorption using water-in-oil-in-water emulsion. Int $J$ Pharm. 1998;172(1-2):189-198.

6. Wei W, Ma GH, Wang LY, Wu J, Su ZG. Hollow quaternized chitosan microspheres increase the therapeutic effect of orally administered insulin. Acta Biomater. 2010;6(1):205-209.
7. Fonte P, Araujo F, Silva C, et al. Polymer-based nanoparticles for oral insulin delivery: revisited approaches. Biotechnol Adv. 2015;33(6 Pt 3): 1342-1354.

8. Lim HP, Tey BT, Chan ES. Particle designs for the stabilization and controlled-delivery of protein drugs by biopolymers: a case study on insulin. J Control Release. 2014;186:11-21.

9. Mukhopadhyay P, Mishra R, Rana D, Kundu PP. Strategies for effective oral insulin delivery with modified chitosan nanoparticles: a review. Prog Polym Sci. 2012;37(11):1457-1475.

10. Pan Y, Li YJ, Zhao HY, et al. Bioadhesive polysaccharide in protein delivery system: chitosan nanoparticles improve the intestinal absorption of insulin in vivo. Int J Pharm. 2002;249(1-2):139-147.

11. George M, Abraham TE. Polyionic hydrocolloids for the intestinal delivery of protein drugs: alginate and chitosan - a review. J Control Release. 2006;114(1):1-14.

12. Wang LY, Gu YH, Zhou QZ, Ma GH, Wan YH, Su ZG. Preparation and characterization of uniform-sized chitosan microspheres containing insulin by membrane emulsification and a two-step solidification process. Colloids Surf B Biointerfaces. 2006;50(2):126-135.

13. Prego C, Torres D, Fernandez-Megia E, Novoa-Carballal R, Quiñoá E, Alonso MJ. Chitosan-PEG nanocapsules as new carriers for oral peptide delivery. J Control Release. 2006;111(3):299-308.

14. Mao S, Bakowsky U, Jintapattanakit A, Kissel T. Self-assembled polyelectrolyte nanocomplexes between chitosan derivatives and insulin. J Pharm Sci. 2006;95(5):1035-1048.

15. Sonvico F, Cagnani A, Rossi A, et al. Formation of self-organized nanoparticles by lecithin/chitosan ionic interaction. Int J Pharm. 2006;324(1):67-73.

16. Gerelli Y, Di Bari MT, Deriu A, et al. Structure and organization of phospholipid/polysaccharide nanoparticles. J Phys Condens Matter. 2008;20(10):104211.

17. Hafner A, Lovric J, Voinovich D, Filipovic-Grcic J. Melatonin-loaded lecithin/chitosan nanoparticles: physicochemical characterisation and permeability through Caco-2 cell monolayers. Int J Pharm. 2009;381(2): 205-213.

18. Senyigit T, Sonvico F, Barbieri S, Ozer O, Santi P, Colombo P. Lecithin/ chitosan nanoparticles of clobetasol-17-propionate capable of accumulation in pig skin. J Control Release. 2010;142(3):368-373.

19. Bhatta RS, Chandasana H, Chhonker YS, et al. Mucoadhesive nanoparticles for prolonged ocular delivery of natamycin: in vitro and pharmacokinetics studies. Int J Pharm. 2012;432(1-2):105-112.

20. Ozcan I, Azizoglu E, Senyigit T, Ozyazici M, Ozer O. Enhanced dermal delivery of diflucortolone valerate using lecithin/chitosan nanoparticles: in-vitro and in-vivo evaluations. Int J Nanomed. 2013;8:461-475.

21. Chhonker YS, Prasad YD, Chandasana H, et al. Amphotericin-B entrapped lecithin/chitosan nanoparticles for prolonged ocular application. Int J Biol Macromol. 2015;72:1451-1458.

22. Barbieri S, Sonvico F, Como C, et al. Lecithin/chitosan controlled release nanopreparations of tamoxifen citrate: loading, enzyme-trigger release and cell uptake. J Control Release. 2013;167(3):276-283.

23. Peng Q, Zhang ZR, Sun X, Zuo J, Zhao D, Gong T. Mechanisms of phospholipid complex loaded nanoparticles enhancing the oral bioavailability. Mol Pharm. 2010;7(2):565-575.

24. Shi K, Cui F, Yu Y, Zhang L, Tao A, Cun D. Preparation and characterization of a novel insulin-phospholipid complex. Asian J Pharm Sci. 2006;1(3-4):168-174.

25. Zhou C, Xia X, Liu Y, Li L. The preparation of a complex of insulinphospholipids and their interaction mechanism. J Pept Sci. 2012; 18(9):541-548

26. Song L, Zhi ZL, Pickup JC. Nanolayer encapsulation of insulin-chitosan complexes improves efficiency of oral insulin delivery. Int J Nanomed. 2014;9:2127-2136.

27. Tan Q, Liu W, Guo C, Zhai G. Preparation and evaluation of quercetinloaded lecithin-chitosan nanoparticles for topical delivery. Int J Nanomed. 2011;6:1621-1630.

28. Rampino A, Borgogna M, Blasi P, Bellich B, Cesaro A. Chitosan nanoparticles: preparation, size evolution and stability. Int J Pharm. 2013; 455(1-2):219-228. 
29. Jose S, Fangueiro JF, Smitha J, et al. Predictive modeling of insulin release profile from cross-linked chitosan microspheres. Eur J Med Chem. 2013;60:249-253.

30. Li X, Qi J, Xie Y, et al. Nanoemulsions coated with alginate/chitosan as oral insulin delivery systems: preparation, characterization, and hypoglycemic effect in rats. Int J Nanomed. 2013;8:23-32.

31. Omid NJ, Babanejad N, Amini H, et al. Preparation and characterization of novel derivatives of chitosan and trimethyl chitosan conjugated with dipeptides and vitamin B12 as candidates for oral delivery of insulin. J Polym Res. 2014;21(8):1.

32. Cui F, Shi K, Zhang L, Tao A, Kawashima Y. Biodegradable nanoparticles loaded with insulin-phospholipid complex for oral delivery: preparation, in vitro characterization and in vivo evaluation. $J$ Control Release. 2006;114(2):242-250.
33. Chaturvedi K, Ganguly K, Nadagouda MN, Aminabhavi TM. Polymeric hydrogels for oral insulin delivery. J Control Release. 2013;165(2): 129-138.

34. Jain D, Panda AK, Majumdar DK. Eudragit S100 entrapped insulin microspheres for oral delivery. AAPS PharmSciTech. 2005;6(1): E100-E107.

35. Wu ZM, Zhou L, Guo XD, et al. HP55-coated capsule containing PLGA/RS nanoparticles for oral delivery of insulin. Int J Pharm. 2012; 425(1-2):1-8.
International Journal of Nanomedicine

\section{Publish your work in this journal}

The International Journal of Nanomedicine is an international, peerreviewed journal focusing on the application of nanotechnology in diagnostics, therapeutics, and drug delivery systems throughout the biomedical field. This journal is indexed on PubMed Central, MedLine, CAS, SciSearch $®$, Current Contents $® /$ Clinical Medicine,

\section{Dovepress}

Journal Citation Reports/Science Edition, EMBase, Scopus and the Elsevier Bibliographic databases. The manuscript management system is completely online and includes a very quick and fair peer-review system, which is all easy to use. Visit http://www.dovepress.com/ testimonials.php to read real quotes from published authors.

Submit your manuscript here: http://www.dovepress.com/international-journal-of-nanomedicine-journal 